\section{Severity of depression and anxi- ety in patients with alopecia areata in Bandar Abbas, Iran}

\author{
Shahram Baghestani, ${ }^{1}$ Shahram Zare, ${ }^{2}$ \\ Seyed Hamzeh Seddigh ${ }^{3}$ \\ Departments of ${ }^{1}$ Dermatology, \\ ${ }^{2}$ Community Medicine, ${ }^{3}$ Psychiatry, School \\ of Medicine, Hormozgan University of \\ Medical Sciences, Bandar Abbas, Iran
}

\section{Abstract}

Alopecia areata (AA) is a chronic disease which esthetic outcomes may result in deep effects on mental disorders of patients. In this case-control study, we compared the mental health of 68 patients diagnosed with AA with 68 healthy individuals using Hamilton anxiety and depression rating scales. There were significant differences between the case and control groups regarding the prevalence of anxiety and depression. The means of anxiety scores in cases and control group were $12.76 \pm 7.21 \mathrm{vs}$ $8.54 \pm 6.37, \mathrm{P}=0.003$. Likewise, the means of depression scores for the groups were $12.84 \pm 4.03$ vs $6.22 \pm 4.95, \mathrm{P}=0.001$. Furthermore, patients with AA were exposed to depression approximately five times and to anxiety about three times more than normal people. Our study revealed a high prevalence of anxiety and depression in AA patients. Dermatologists should pay more attention on psychological effect of the disease on the patients.

\section{Introduction}

Alopecia areata (AA) is considered as a chronic and common inflammatory disease that affects mostly hair follicles along with nails involvement in few cases. This disorder, which is characterized by the sudden hair loss on the scalp or other hair bearing areas, is seen in both sexes, at all ages.

Various factors, including genetic, endocrine, immunologic, infectious, spiritual, and psychological factors are supposed to have a role in its pathogenesis. ${ }^{1}$

The role that psychological factors play in the pathogenesis of AA has been the subject of discussion for decades. Numerous studies have been done in relation to this matter that has resulted in controversial outcomes. ${ }^{2-14}$ The objective of our study was to investigate the severity of anxiety and depression in patient with AA.

\section{Materials and Methods}

This case-control study, was carried out on 68 confirmed patients ( $>18$ years old) suffering from AA (on scalp) referred to the skin clinic, affiliated to Hormozgan University of Medical Sciences (Bandar Abbas, Iran). Sixty eight healthy people from general population selected as control, matched with the case group by age, sex and the level of education. Informed consent was obtained, and according to severity of disease on scalp, patients were divided into five groups (S1: 0-25\%, S2: 2649\%, S3: 50-74\%, S4: $75-99 \%$ and S5: $100 \%)$ based on Olsen/Canfield criteria. ${ }^{14}$ According to the location of the disease onset, the patients were classified into four groups as follow: temporal, occipital, frontal and multifocal. Finally, from the view point of education level, the participants were grouped as primary, secondary and higher education.

Severity of anxiety and depression were assessed respectively by the use of Persian versions of Hamilton anxiety rating scale (HamA) and Hamilton depression rating scale (Ham-D). ${ }^{15,16}$

All statistical analyses were performed by means of SPSS-16 (SPSS Inc. Chicago, IL, USA). T-student, Analysis of variance and ChiSquare tests were used to compare the groups. Logistic regression was applied to estimate odds ratios of the disease for each of the outcome variables. P-value $<0.05$ was considered as significant.

\section{Results}

Seventy-two percent of both cases and controls were male. The age range for the cases was from 19 to 64 with average of $35.4 \pm 7.6$ years and for the control group was 20 to 63 with average of $33.8 \pm 8.1$ years. When compared the two groups, no significant difference regarding age and sex could be observed. The mean of disease duration in men and women was 22.7 and 20.8 months, respectively.

Based on Ham-A, there were 32 cases (47\%) suffering from anxiety. The mean of anxiety scale in case group was significantly more than that of the control group $(12.76 \pm 7.21 \mathrm{vs}$ $8.54 \pm 6.37 ; \mathrm{P}=0.003)$. Moreover, according to Ham-D, there were 38 cases (56\%) suffering from a degree of depression. The mean of depression scale in case group was significantly more than the control group $(12.84 \pm 4.03 \mathrm{vs}$ $6.22 \pm 4.95 ; \mathrm{P}=0.001)$. Table 1 demonstrates the frequency of anxiety and depression severity in case and control groups based on sex. The severity of anxiety and depression was significant in both the groups $(\mathrm{P}<0.05)$.

Odds ratios (OR) were calculated using a
Correspondence: Shahram Zare, Department of Community Medicine, Hormozgan University of Medical Sciences, Jomhoori Blvd. Mohammadi Hospital, Education Department, 7919915519 Bandar Abbas, Iran

Tel.: +98.917.161.3924 - Fax: +98.763.333.7618.

E-mail: shzare159@gmail.com

Key words: Alopecia areata; depression; anxiety; Iran.

Acknowledgements: the authors would like to thank the research and information technology department of Hormozgan University of Medical Sciences for funding the research.

Contributions: SB, SZ, references search, manuscript writing; SHS, data collecting; SZ, data analyzing; SB, SZ, SHS, manuscript reviewing.

Conflict of interest: the authors declare no potential conflict of interest.

Funding: the study was financially supported by vice-chancellor for research and information technology, Hormozgan University of Medical Sciences.

Received for publication: 7 June 2015. Revision received: 1 October 2015.

Accepted for publication: 2 October 2015.

This work is licensed under a Creative Commons Attribution NonCommercial 3.0 License (CC BYNC 3.0).

CC Copyright S. Baghestani et al., 2015

Licensee PAGEPress, Italy

Dermatology Reports 2015; 7:6063

doi:10.4081/dr.2015.6063

bivariate logistic regression model in order to measure the strength of the relationship between the disease and depression or anxiety. Results show that, patients with AA are exposed to depression approximately five times more than normal people $(\mathrm{OR}=4.48$; 95\%CI: 2.12-9.44). However, the odds ratio of anxiety originated from AA was $2.72(95 \% \mathrm{CI}$ : 1.30-5.68).

Table 1 shows the severity of anxiety and depression in the case and control groups based on sex. A degree of anxiety was observed in $44.9 \%$ of men and $52.7 \%$ of women in case group compared with $16.3 \%$ of men and $42.1 \%$ of women in control group. Moreover, $53.1 \%$ of men and $63.2 \%$ of women in case group and $18.4 \%$ of men and $31.6 \%$ of women in control group were suffering from depression. Mean scores for anxiety and depression were significantly different in the case and control groups $(\mathrm{P}=0.003)$.

In this study, comparison of prevalence, severity of anxiety, and severity of depression between the two groups revealed no significant difference with respect to age, whereas a 
highly significant difference was observed from the education level's point of view. Table 2 shows the prevalence of anxiety of case and control groups based on education level.

The prevalence of anxiety and depression was significantly different between the case and control groups considering the education level in two levels of primary and secondary education (P-values<0.05) (Table 2).

Regarding the means of anxiety and depression when it comes to the level of severity of the disease (Table 3), the results showed that there was significant difference in anxiety and depression mean scores based on the severity of disease (P-values<0.05).

No significant relationship was observed concerning the mean of anxiety and depression and the onset location of the disease. Likewise, there was no significant correlation between duration of the disease, and the severity of anxiety or the severity of depression.

\section{Discussion}

The purpose of this paper is to investigate the psychological effects of alopecia areata. Alopecia areata, which can be considered as a triggering factor in psychological disorders such as anxiety and depression, have been the subject of many previous studies, with contradictory results. ${ }^{5-12}$ The present study revealed that psychological disorders are prevalent in patients with AA; this is in accordance with the results of other studies carried out on adults with AA..$^{8-10,13,14}$ However, Yasemi and Colon in their studies had observed no difference between case and control groups. ${ }^{6,11}$

Despite the fact that AA patients compared to the general population are more suffering from anxiety and depression, the disorder may not confirm the etiological role of psychological factors, it suggests that anxiety and depression are named as comorbid mental disorders.
This aspect is matched with the perspective of Sadock et al. ${ }^{17}$ about the role of psychological factors involved in this disease. They stated that, although the role of stressful events in onset and aggravation of AA is not clear, still comorbid mental disorders have been seen. Psychological diagnoses such as substantial depression, generalized anxiety disorder and paranoid disorders have been reported in

Table 1. Frequency of anxiety and depression in case and control groups based on sex.

\begin{tabular}{lccc} 
Mental disorder & Case $(n=68), \mathbf{N}(\%)$ & Control $(n=68), \mathbf{N}(\%)$ & P-value \\
& \multicolumn{3}{c}{ Anxiety } \\
Mean \pm SD & $12.76 \pm 4.21$ & $8.54 \pm 3.37$ & 0.003 \\
Male & $22(44.9)$ & $8(16.3)$ & 0.001 \\
Weak & $11(22.4)$ & $5(10.2)$ & \\
Mild & $9(18.4)$ & $3(6.1)$ & \\
Severe & $2(4.1)$ & $0(0.0)$ & 0.022 \\
\hline Female & $10(52.7)$ & $8(42.1)$ & \\
Weak & $3(15.8)$ & $5(26.3)$ & \\
Mild & $4(21.1)$ & $2(10.5)$ & 0.003 \\
Severe & $3(15.8)$ & $1(5.3)$ & 0.001 \\
& & & \\
Mean \pm SD & $12.84 \pm 4.03$ & $6.22 \pm 1.95$ & \\
Male & $26(53.0)$ & $9(18.4)$ & \\
Weak & $6(16.3)$ & $2(4.1)$ & 0.001 \\
Mild & $7(14.3)$ & $0(0.0)$ & \\
Severe & $11(22.4)$ & $7(14.3)$ & \\
\hline Female & $12(63.2)$ & $6(31.3)$ & \\
Weak & $3(15.8)$ & $3(15.8)$ & \\
Mild & $4(21.1)$ & $3(15.8)$ & \\
Severe & $5(26.3)$ & & \\
\hline
\end{tabular}

SD, standard deviation.

Table 2. Prevalence of anxiety and depression in the patients based on education level.

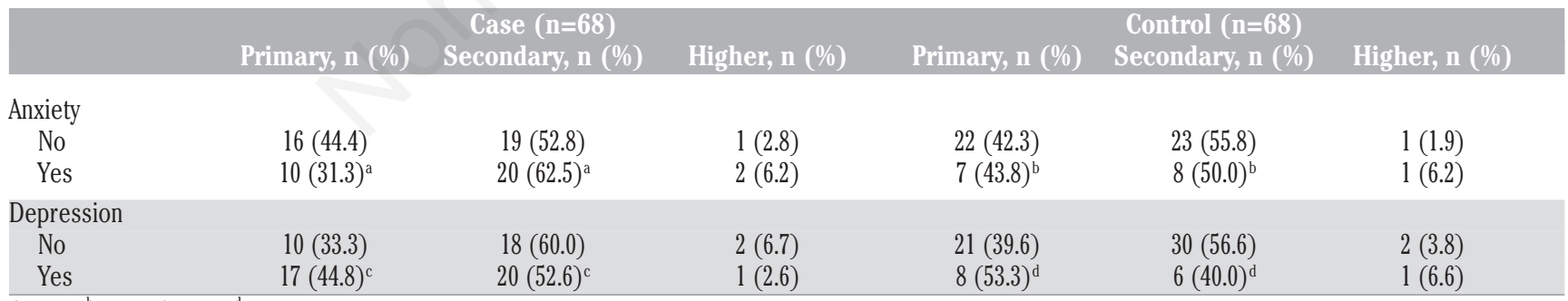

${ }_{\mathrm{a}} \mathrm{P}=0.012,{ }_{\mathrm{P}}=0.038,{ }^{\mathrm{C}} \mathrm{P}=0.023,{ }_{\mathrm{P}}=0.028$

Table 3. Mean of anxiety and depression scores of the patients based on severity of the disease.

\begin{tabular}{lccc} 
Severity of the disease & $\mathrm{n}(\%)$ & Anxiety, ${ }^{\mathrm{a}}$ mean \pm SD & Depression, ${ }^{\mathrm{b}}$ mean \pm SD \\
S1 & $25(36.8)$ & $11.45 \pm 4.07$ & $11.55 \pm 5.35$ \\
S2 & $22(32.3)$ & $12.89 \pm 4.33$ & $11.86 \pm 6.41$ \\
\hline S3 & $10(14.7)$ & $14.15 \pm 5.27$ & $12.98 \pm 4.32$ \\
S4 & $6(8.8)$ & $14.21 \pm 3.51$ & $14.17 \pm 2.42$ \\
S5 & $5(7.4)$ & $15.19 \pm 3.35$ & $13.14 \pm 3.12$ \\
Total & $68(100)$ & $12.76 \pm 4.21$ & $12.84 \pm 4.03$
\end{tabular}

SD, standard deviation. ${ }^{\mathrm{a}} \mathrm{P}=0.038, \mathrm{bP}=0.041$. 
many cases. ${ }^{17}$

In our study, like Firooz who reported no relationship between the concerns of patients about their disease and their age, ${ }^{18}$ there was no significant difference between prevalence, severity of anxiety and severity of depression in AA patients and the control group when discussing the age. Whereas, Safa reported that the number of severe cases of depression in 16-25 age-group was more than other agegroups. ${ }^{9}$ This difference might be due to differences in age groups or lack of control group in Safa study. ${ }^{9}$

In our study, females were differentiated with males by higher prevalence of anxiety and depression. These findings are in accordance with results of other studies that indicated a higher prevalence of psychiatric disorders among females with AA., ${ }^{1,9}$ The researcher suggested that it was due to higher rate of aesthetic stress for women. Nevertheless, Firooz found no relationship between patients' concerns about the outcomes of the disease and sex. ${ }^{18}$ Definitely, to study the role of sex in development of mental disorders in $\mathrm{AA}$ patients, considerable number of cases is required to be studied and investigated.

Our findings also showed a significant statistical difference between those with primary and secondary education level concerning the prevalence of anxiety and depression. Nevertheless, Safa and Firooz showed that there was not a significant relationship between education level and psychological disorders in the patients. ${ }^{9,18}$

In line with many published studies, our findings revealed a significant difference in average anxiety and depression scores based on extent of the disease. ${ }^{1,19}$ This finding may confirm that stressful events in life can be considered as an accelerating factor for the onset of $\mathrm{AA}$, as Manolache in a study on $114 \mathrm{AA}$ patients concluded that trauma and acute anxiety were the most common causes of $\mathrm{AA}^{20}$

No significant relationship was observed between neither the onsite location of the disease and anxiety nor the onsite location and depression. Safa also reported the same results. ${ }^{9}$

In our patients, there was not a significant relationship between average anxiety and depression scores and duration of the disease. Safa also did not find relationship between psychological disorders and duration of disease. ${ }^{9}$ However, Firooz pointed out the direct relationship between increased patient's concerns with that of the increased duration of disease. ${ }^{18}$

\section{Conclusions}

Although the fact that AA patients compared to the general population are more suffering from anxiety and depression disorders, but it may not confirm the etiological role of psychological factors, it suggests anxiety and depression as comorbid mental disorders.

\section{References}

1. Sellami R, Masmoudi J, Ouali U, et al. The relationship between alopecia areata and alexithymia, anxiety and depression: a case-control study. Indian J Dermatol 2014;59:421-8.

2. Chu SY, Chen YJ, Tseng WC, et al. Psychiatric comorbidities in patients with alopecia areata in Taiwan: a case control study. Br J Dermatol 2012;166:525-31.

3. Ghanizadeh A, Ayoobzadeh A. A review of psychiatric disorders comorbidities in patients with alopecia areata. Int $\mathbf{J}$ Trichology 2014;6:2-4.

4. Alfani S, Antinone V, Mozzetta A, et al, Psychological status of patients with alopecia areata. Acta Derm Venereol 2012;92:304-6.

5. Schmitt JV, Riberio CF, Souza FH, at al. Hair loss perception and symptoms of depression in female outpatients attending a general dermatology clinic. An Bras Dermatol 2012;87:412-7.

6. Yasemi MT, Mansouri P, Personal characteristics and anxiety severity in patients with regional hair loss. Kerm Med Sci J 1994;2:65-70.

7. Gulec AT, Tanriverdi N, Duru C, et al. The role of psychological factors in alopecia areata and the impact of the disease on the quality of life. Int $\mathrm{J}$ Dermatol 2004;43:352-6.

8. Kose 0, Sayar K, Ebrinc S. Psychometric assessment of alopecia areata patients before and after dermatological treatment. Bull Clin Psychopharmacol 2000;10:21-5.
9. Safa M, Jabreili R, Moamen-Nasab M. Depression and anxiety frequency in patients with alopecia areata, referred to dermatology and psychology clinics of Khorram Abad. Yafteh 2007;9:33-7.

10. Kalafi Y, Mousavi-nasab M, Tobaei S, et al. The role of psychiatric disorder in paitient with Alopecia Areata. Iran J Med Sci 1993;18:21-5.

11. Colón EA, Popkin MK, Callies AL, et al. Lifetime prevalence of psychiatric disorders in patients with alopecia areata. Compr Psychiatry 1991;32:245-51.

12. Sayar K, Köse 0, Ebrinç S, Cetin M. Hopelessness, depression and alexithymia in young Turkish soldiers suffering from alopecia areata. Dermatol Psychosomat 2001;2:12-5.

13. Diaz-Atienza F, Gurpegui M. Environmental stress but not subjective distress in children or adolescents with alopecia areata. J Psychosom Res 2011;71:102-7.

14. Olsen EA, Hordinsky MK, Price VH, et al. Alopecia areata investigational assessment guidelines-part II. National Alopecia areata Foundation. J Am Acad Dermatol 2004;51:440-7.

15. Hamilton M. The assessment of anxiety states by rating. $\mathrm{Br} \mathrm{J}$ Med Psychol 1959;32:50-5.

16. Williams JBW. A structured interview guide for the Hamilton Depression Rating Scale. Arch Gen Psychiatry 1988;45:742-7.

17. Sadock BJ, Sadock VA, Kaplan HI. Comprehensive textbook of psychiatry. 8th ed. Vol. 3. Philadelphia: Williams \& Wilkins; 2005.

18. Firooz A, Firoozabadi MR, Ghazisaidi B, Dowlati Y. Concepts of patients with alopecia areata about their disease. BMC Dermatol 2005;5.

19. Ruiz-Doblado S, Carrizosa A, GarciaHernandez MJ. Alopecia areata: psychiatric comorbidity and adjustment to illness. Int J Dermatol 2003;42:434-7.

20. Manolache L, Benea V. Stress in patients with alopecia areata and vitiligo. J Eur Acad Dermatol Venereol 2007;21:921-8. 